\title{
Resiliencia en adolescentes institucionalizados. Intervención con terapia de juego Gestalt
}

\section{Resilience in institucionalized adolescences. A Gestalt play therapy intervention}

\author{
Abril Pacheco Patrón \\ Elia M. Escoffié Aguilar \\ Jorge Navarrete Centeno \\ Universidad Autónoma de Yucatán (UADY)
}

\section{Resumen}

El objetivo de este estudio es conocer si un programa de intervención en terapia de juego Gestalt, favorece la capacidad de resiliencia en adolescentes institucionalizados. La muestra estuvo conformada por tres participantes, varones, de edades entre 13 y 16 años. El instrumento empleado en el pre y post test, fue el Cuestionario de Resiliencia (fuerza y seguridad personal) de González Arratia et al. (2008), el cual consta de 30 reactivos. El programa de intervención constó de 20 sesiones de duración aproximada de 45 minutos. Los resultados interpretados de manera individual indican que los niveles de resiliencia aumentaron después de la intervención en todos los factores, no obstante, el aumento de la resiliencia no fue estadísticamente significativo. Se discuten los resultados en función del tamaño de la muestra; no se puede concluir que la terapia de juego Gestalt contribuye en el fortalecimiento de la capacidad de resiliencia para esta muestra de adolescentes.

Palabras clave: estudio de caso clínico, resiliencia, adolescentes, institucionalización, Gestalt.

\begin{abstract}
The objective of this study is to know if an intervention program in Gestalt play therapy, favors the capacity of resilience in institutionalized adolescents. The sample consisted of three participants, males, aged between 13 and 16 years. The instrument used in the pre and post-test was the Resilience Questionnaire (strength and personal security) of González Arratia et al. (2008), which consists of 30 reagents. The intervention program consisted of 20 sessions lasting approximately 45 minutes. Indi-

Nota del autor

Abril Pacheco Patrón, Facultad de Psicología, Universidad Autónoma de Yucatán (UADY).

La correspondencia en relación con este artículo debe dirigirse a Abril Pacheco Patrón, Facultad de Psicología, UADY, carretera Mérida-Tizimin, km1, colonia Centro, C.P. 97000, Cholul. Mérida, Yucatán, México.

Dirección electrónica: abrilpacheco@gmail.com
\end{abstract}


vidually interpreted results indicate that resilience levels increased after intervention on all factors, however, the increase in resilience was not statistically significant. Results are discussed in terms of sample size; It can't be concluded that Gestalt play therapy contributes to strengthening resilience for this sample of adolescents.

Keywords: clinical case study, resilience, adolescents, institutionalization, Gestalt.

Dependiendo de las características del contexto local, las personas en situación de pobreza, con problemas de salud crónicos, los niños y adolescentes expuestos al maltrato, al abandono o al abuso de sustancias, los grupos minoritarios, las poblaciones indígenas, las personas de edad avanzada, etcétera., pueden correr un riesgo significativamente mayor de sufrir problemas de salud mental (Organización Mundial de la Salud, 2013). La etapa del desarrollo puede ser una situación de riesgo, según Tarín y Navarro (2006), "la etapa adolescente se considera un periodo crítico en el inicio y afianzamiento de conductas de riesgo" (p. 35).

El periodo de la adolescencia, que implica per se un periodo de transición, con matices y cambios cognitivos, físicos y emocionales, demanda en la población infanto-juvenil el uso de estrategias de afrontamiento que les permitan adaptarse mejor a la gama de eventos vitales estresantes propios de su edad (Pardo, Sandoval, \& Umbarila, 2004, como se citó en RestrepoRestrepo, Alpi, \& Quiceno, 2011). "Existe una gran variabilidad individual respecto a la influencia de los factores estresantes" (Antonovsky, 1987; Lazarus, \& Folkman, 1984;
Kobasa, 1979, como se citó en Sanz Rodríguez et al., 2009). Por ejemplo, un evento estresante en la vida de la persona (el abandono, maltrato o negligencia en la infancia), no se correlaciona con una enfermedad mental en el futuro, por el contrario, fortalecer al ser humano a través de una intervención integrativa multimodal, contribuye a desarrollar las habilidades necesarias para afrontar situaciones adversas sin desarrollar una psicopatología (Rocha-Arragoniz, 2012).

Si la adolescencia es un riesgo per se, los adolescentes institucionalizados tienen una mayor probabilidad de presentar problemas en su desarrollo, dado que se convierten en niños o adolescentes en riesgo de maltrato y/o abandono (Barcelata, 2015). La institucionalización y el maltrato han sido variables que han sido estudiadas para conocer su relación con la resiliencia. Los estudios de la resiliencia iniciaron con Emmy Werner (1989), quien realizó una observación longitudinal durante 32 años de 698 niños y niñas hawaianos en la isla de Kauai, encontró que algunos de esos niños que habían nacido y crecido en medio de familias con diferentes problemas, crecían convirtiéndose en adultos felices, bien adaptados y creativos, por ende, concluyó que hubo casi $80 \%$ de casos con 
evoluciones favorables (Barudy, \& Dantagnan, 2007).

En un estudio realizado con niños de entre 8 y 11 años no se encontraron diferencias estadísticamente significativas de la resiliencia en niños institucionalizados y no institucionalizados (Gianino-Gadea, 2012). Datos similares se registraron respecto la medición del nivel de resiliencia en mujeres adolescentes institucionalizadas en un orfanato; en el estudio se encontraron niveles altos en los diferentes factores de resiliencia, niveles altos en comparación con la población en general (Pérez-Ruano, 2014). En otro estudio cualitativo se encontró que los adultos cuya infancia transcurrió en el interior de un hogar de menores, pueden transmitir a su núcleo familiar un estilo de vida resiliente (Araya-Escobar, 2014).

Los adolescentes o niños resilientes se caracterizan por un coeficiente de inteligencia mayor, menor búsqueda de novedad y de afiliación con pares delincuentes (Fergusson, \& Lynskey, 1996), y además de mayores habilidades de auto-regulación y autoestima (Buckner, Mezzacappa, \& Beardslee, 2003). En un estudio comparativo de niños maltratados y no maltratados, se encontró que los primeros eran más prosociales y menos agresivos, tenían menos conductas disruptivas y menos peleas que los niños no maltratados (Flores, Cicchetti, $\&$ Rogosch, 2005). Un estudio sugiere que la resiliencia es un atributo de la personalidad $y$ no requiere de la presencia de la adversidad (Cicchetti, \& Rogosch, 1997). Con base en la misma línea de estudio en niños maltratados o abandonados, existe evidencia de que las mujeres tienen mayores probabilidades de tener conductas resilientes en comparación con los hombres (Mcgloin, \& Spatz-Widom, 2001).

No existe un consenso para medir y supervisar las intervenciones que se realizan con el fin de fortalecer la resiliencia (Bené, Frankenberger, \& Nelson, 2015). En un programa de intervención para facilitar técnicas en conductas resilientes se tomaron indicadores como bajos niveles de ansiedad y percepción de estrés, así como un aumento de satisfacción y compromiso de la vida como medidas de efectividad de la intervención (Kees, \& Rosenblum, 2015). En otros estudios la resiliencia, es un factor importante el manejo del estrés (Hou et al., 2016; Steinhardt, \& Dolbier, 2008) y puede tomarse también como un indicador.

Se sugiere que en un proceso de intervención para aumentar la resiliencia en niños, se identifiquen los factores de protección, utilizar técnicas como el juego libre o el ensayo conductual, así como el entrenamiento en técnicas de relajación y autocontrol (Alvord, \& Grados, 2005).

\section{Psicoterapia de juego Gestalt}

La terapia de juego ha ido tomando gran relevancia en los últimos años, sobre todo cuando la intervención se centra en niños. En estos tipos de intervención se dice que el juego es el lenguaje 
del niño y los juguetes sus palabras (Landreth, 2002). La asociación para la terapia de juego, en 1997 (APT, por sus siglas en inglés), la definió como "el uso sistemático de un modelo teórico para establecer un proceso interpersonal en el que terapeutas capacitados utilizan los poderes terapéuticos del juego para ayudar a sus clientes a prevenir y resolver dificultades psicosociales y alcanzar un desarrollo y crecimiento óptimos" (p. 7).

El juego repercute en todas las áreas de desarrollo del niño y adolescente, ayuda a perfeccionar sus habilidades, enriquece el conocimiento de su ambiente y de sí mismo, además de favorecer para una adecuada autoestima y autorregulación (Esquivel-Ancona, 2010). La efectividad de las terapias de juego ha sido muy criticada pero también existen estudios meta-analíticos que ha mostrado su efectividad (Ray, Bratton, Rhine, \& Jones, 2001; Bratton, Ray, Rhine, \& Jones, 2005). La terapia de juego ha mostrado ser efectiva en el tratamiento del estrés que tienen los niños al relacionarse con sus pares (Ray, 2008), así como la auto eficacia (Fall, Balvanz, \& Johnson, 1999).

Se desarrolló un programa de intervención con el objetivo de favorecer la capacidad de resiliencia en adolescentes institucionalizados, a través de intervenciones psicoterapéuticas individuales, con base en la metodología de terapia de juego con enfoque Gestalt. También se planteó la necesidad de medir los niveles de resiliencia en los adolescentes antes y después de la intervención psicoterapéutica.
La intervención psicoterapéutica fue dividida en cuatro fases: de diagnóstico, inicial, intermedia y final, "con características distintivas en cuanto a la función del terapeuta, el trabajo terapéutico, las herramientas utilizadas y los objetivos a lograr" (Fernández-Rodríguez, 2014, p. 26).

Durante la fase de diagnóstico se busca el establecimiento del rapport y la elaboración de un diagnóstico fenomenológico, que puede estar acompañado de instrumentos de medición. A partir del diagnóstico que se planea el proceso terapéutico (Fernández-Rodríguez, 2014). En la fase inicial, "se hace necesario que niños y adolescentes asimilen el estilo de trabajo y desarrollen habilidades de darse cuenta y de contacto, que permitan el libre fluir del proceso terapéutico, validando las emociones y creando la conciencia emocional" (Fernández-Rodríguez, 2014, p. 29). En la fase intermedia, el paciente aprende cómo expresa su sí mismo en el ambiente, por lo cual en esta fase "los juegos y procesos se encaminan a trabajar con las emociones en conflicto, con las introyecciones negativas, con la integración de sus polaridades, con las partes rechazadas del sí mismo" (Fernández-Rodríguez, 2014, p. 30). La fase final es un proceso de interiorización del avance terapéutico y en ella se realizaron las aplicaciones pos-test de la intervención.

Por lo tanto, el objetivo del estudio es conocer si un programa de intervención en terapia de juego Gestalt, favorece la capacidad de resiliencia en adolescentes institucionalizados. 


\section{Método}

\section{Participantes}

Para la selección de los participantes del estudio, se aplicó el instrumento de resiliencia a la población total del albergue (nueve adolescentes), de la cual fueron seleccionados cuatro personas, tomando como criterio de inclusión que obtuvieran un puntaje en la escala total de 3 puntos o menos, de manera tal que quienes obtuvieran arriba de 4 puntos, quedarían excluidos del estudio, por señalar sus resultados un buen nivel de resiliencia.

El estudio se inició con cuatro jóvenes varones, dos de 13 años y dos de 16 años de edad, aunque uno de ellos escapó del albergue durante el periodo en el que se realizó la intervención psicoterapéutica, se concluyó únicamente con tres.

De los cuatro participantes, dos de ellos son originarios de Sonora y los otros dos de otro estado de la República Mexicana, pero dado que en su estado no cuentan con albergues apropiados para ellos, fueron referidos a Yucatán. Asimismo, de los cuatro participantes, tres se encontraban realizando sus estudios de educación secundaria y uno de nivel primaria.

\section{Instrumentos}

El instrumento empleado a manera de pre y pos-test fue el Cuestionario de Resiliencia (Seguridad y Fuerza Personal) de GonzálezArratia, Valdez-Medina, \& Zavala-Borja (2008).
El instrumento está compuesto, en su versión original, por 70 ítems, con cinco opciones de respuesta tipo likert, que van de Siempre, La mayoría de las veces, Indeciso, Algunas veces y Nunca. En el análisis de la confiabilidad se encontró una consistencia interna de 9192. De los resultados del Análisis de Componentes Principales (ACP) con rotación Varimax, se generó la versión del instrumento empleado en la presente investigación, y: que consta de 30 reactivos que se conservaron de los 70 originales, por pasar todas las pruebas del análisis.

Con el ACP se obtienen tres factores, el primero agrupa doce reactivos $(17,18,20,21$, $23,24,25,26,27,28,29$ y 30$)$ y se les denomina factores protectores internos; hacen referencia a los atributos de la propia persona: estima, seguridad y confianza en sí mismo, facilidad para comunicarse.

El segundo factor lo conforman once reactivos (números 1, 4, 5, 6, 10, 11, 12, 13, 14, 15 y 16) y se les denomina factores protectores externos, lo cual hace alusión a las condiciones del medio que actúan y reduce la probabilidad de daños: familia extendida, apoyo de un adulto significativo o integración social o laboral (Munist et al., 1998 como se citó en GóngoraMárquez, 2011).

Por último, el tercer factor se compone de siete reactivos $(2,3,7,8,9,19$ y 22$)$, se le nombra empatía, al referirse a la relación que existe entre el hecho de hacer frente a una experiencia traumática $\mathrm{y}$ que esto propicie o despierte 
en la persona sentimientos de compasión y empatía hacia el sufrimiento de otras personas y promueva conductas altruistas (Vera, 2005 como se citó en Góngora-Márquez, 2011).

\section{Procedimiento}

Se aplica el instrumento de resiliencia a manera de pre y pos-test para evaluar la eficacia del programa de intervención desarrollado a través de 20 sesiones individuales, de 45 minutos de duración aproximada cada una, con periodicidad semanal, el análisis e interpretación de los resultados del instrumento de resiliencia, dado que es una escala tipo likert, se interpretan tomando como referencia la media teórica que es tres, por lo tanto, todas las puntuaciones pos test por debajo de ese valor se consideran abajo de la media y por el contrario, las puntuaciones pos-test mayores a dicho valor se interpretan como por encima de la media.

Se llevó a cabo una prueba $t$ para muestras relacionadas con el fin de conocer si existen diferencias entre la calificación inicial y final de la resiliencia en los dos momentos de la intervención. Debido al tamaño de muestra se realizó un submuestreo mediante técnicas de bootstrap.

\section{Resultados}

Las puntuaciones medias de cada uno de los factores se presentan en la tabla 1. Se muestran tanto la calificación media antes de la intervención (pre) como al finalizar la intervención (post). Se puede observar, en el factor interno, que dos sujetos tuvieron una puntuación por debajo de un media teórica de 3 y sólo una mayor a 3. Asimismo, en el factor externo, una persona tuvo una puntuación muy por arriba de 3 en pre. El sujeto uno tiene las puntuaciones más altas en los tres factores al inicio de la intervención.

Tabla 1

Resultados obtenidos de cada factor del instrumento de resiliencia por cada participante antes (pre) y después (post) de la intervención

\begin{tabular}{ccccccc}
\hline $\mathrm{N}$ & \multicolumn{2}{c}{ Factor interno } & \multicolumn{2}{c}{ Factor externo } & \multicolumn{2}{c}{ Empatía } \\
\hline & Pre & Post & Pre & Post & Pre & Post \\
1 & 3.16 & 3.60 & 4.00 & 4.10 & 3.00 & 3.14 \\
2 & 2.08 & 3.80 & 2.18 & 4.36 & 2.85 & 3.70 \\
3 & 2.50 & 4.58 & 2.27 & 4.72 & 2.14 & 4.43 \\
\hline
\end{tabular}

Para conocer si hubo un aumento de la calificación, se realizó una diferencia entre la calificación del post y del pre para cada factor [post-pre]. Los resultados se presentan de manera numérica en la tabla 2 y de manera visual en la figura 1. Si la diferencia de un factor es positiva $(+)$ se considera una ganancia o aumento de la resiliencia; si la diferencia es negativa ( - ) representa una pérdida o una disminución de la resiliencia al final de la intervención. Los datos se presentan hasta ahora de manera descriptiva. 


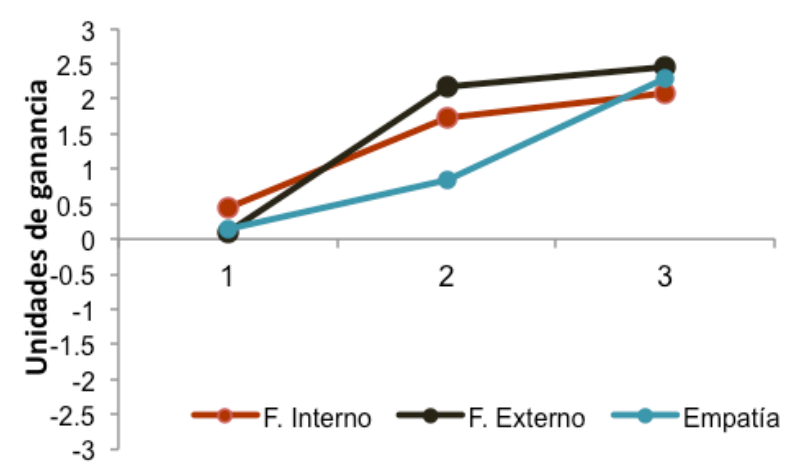

Figura 1. Gráfica de unidades de ganancia por factor.

Tabla 2.

Unidades de ganancia pre y post-test

\begin{tabular}{cccc}
\hline $\mathrm{N}$ & F. Interno & F. Externo & Empatía \\
\hline 1 & 0.44 & 0.1 & 0.14 \\
2 & 1.72 & 2.18 & 0.85 \\
3 & 2.08 & 2.45 & 2.29 \\
\hline
\end{tabular}

$\mathrm{Al}$ observar la figura $1 \mathrm{y}$ la tabla 2 , se puede notar que las diferencias son positivas, eso indica que existe un aumento de la resiliencia; las unidades de ganancia se presentan en la figura 1. El sujeto uno es la persona que tuvo el menor aumento y la persona tres es quien tuvo un mejor aprovechamiento de la intervención. No obstante, al analizar la tabla 1 se puede notar que la persona uno registró una calificación alta cuando inició la intervención y eso puede explicar el porqué su ganancia fue menor.

Para abordar el objetivo planteado de la presente investigación se analizó el efecto de la intervención mediante una prueba de bootstrap para muestras apareadas. Los resultados se presentan en la tabla 3. Las medias hacen referencia a una media de las diferencias con un intervalo de confianza de $95 \%$. Los valores $p$ de la diferencia de medias no fueron significativos.

Tabla 3.

Prueba bootstrap para Prueba de muestras emparejadas

\begin{tabular}{llcc}
\hline \multicolumn{1}{c}{ Factor } & & Media & Valor $\mathrm{p}$ \\
\hline Factor interno & Post-pre & 1.4133 & $.259^{\mathrm{b}}$ \\
Factor externo & Post-pre & 1.5767 & $.259^{\mathrm{b}}$ \\
Empatía & Post-pre & 1.0933 & $.247^{\mathrm{b}}$ \\
\hline
\end{tabular}

Los resultados de la tabla 3 parecen indicar que no existe un aumento de la resiliencia después de la intervención, no obstante, al considerar a la media teórica de 3 se realizó una prueba $t$. En contraste con la tabla 3, los resultados son significativos para el factor interno y externo, pero no para la empatía. Dichos resultados sugieren que los factores internos y externos registraron una puntuación por arriba de la media teórica al finalizar el proceso de intervención.

Tabla 4.

Prueba t para una muestra con un valor de prueba de 3

\begin{tabular}{lccccc}
\hline \multicolumn{1}{c}{$\begin{array}{c}\text { Puntación } \\
\text { POST }\end{array}$} & Media & DE & t & gl & P \\
\hline F. Interno & & & & & \\
F. Externo & 4.99 & 0.52 & 3.32 & 2 & .080 \\
Empatía & 3.76 & 0.31 & 7.71 & 2 & .016 \\
\hline
\end{tabular}




\section{Discusión}

Los resultados tienen matices que valen la pena ser analizados: 1) los resultados significativos cuando se comparan con la media teórica y los resultados cuando se evalúa la efectividad del taller como un modelo de pre y pos-test. 2) los resultados analizados desde el tamaño de la muestra y las inferencias a la población. 3) los resultados a la luz de la literatura del marco teórico.

Con base en el objetivo de la investigación, el análisis de bootstrap para muestras emparejadas sugiere que la intervención no tuvo efecto alguno en la resiliencia.

Existen varios elementos que pueden ayudar a explicar estos resultados: el tamaño de muestra para el análisis es muy pequeña para poder determinar la efectividad del programa, por otro lado, de los tres sujetos que componen la muestra uno de ellos inició el programa de intervención con una puntuación mayor a sus otros compañeros y al final del programa no mostró un aumento en sus calificaciones de resiliencia en sus factores internos (ver figura 1).

Es importante mencionar que el estudio de Mcgloin y Spatz-Widom, (2001) menciona que existe evidencia según la cual las mujeres tienen mayores probabilidades de tener conductas resilientes en comparación con los hombres, por otro lado, en un estudio realizado con niños de entre 8 y 11 años no se encontraron diferencias estadísticamente significativas de la resiliencia entre niños institucionalizados y no institucionalizados (Gianino-Gadea, 2012). Por lo que, independientemente de la intervención, pareciera que utilizar un modelo de grupo de control para analizar los resultados no es una buena opción.

Pese a los resultados en apariencia contradictorios entre el análisis de muestras pareadas y los resultados de la prueba t para una muestra, se considera que pueden ser complementarios. El programa de intervención quizá no tuvo el efecto deseado o esperado, pero al menos es significativamente mayor a una media teórica esperada, es decir, es significativamente mayor a una puntuación teórica de la población en general, según la escala.

Los resultados encontrados pueden ser tratados y analizados con cautela y no es posible concluir por el tamaño de muestra que el programa haya tenido o no un aumento o disminución de la resiliencia. No obstante, dentro de los factores evaluados, el factor de empatía fue el factor con las puntuaciones más bajas para los tres participantes.

\section{Referencias}

Alvord, M. K., \& Grados, J. J. (2005). Enhancing Resilience in Children: AProactive Approach. Professional Psychology: Research and Practice, 36(2), 238-245.

Araya Escobar, A. (2014). Adultos Resilientes,

Cuya Infancia y/o Adolescencia Fue Institucionalizada. Tesis para optar al Título de Asistente Social. Santiago de Chile: Escuela de Trabajo Social, Universidad de Academia de Humanismo Cristiano. 
Barcelata-Eguiarte, B. E. (2015). Adolescentes en riesgo. Una mirada a partir de la resiliencia. México: Manual Moderno.

Barudy, J., \& Dantagnan, M. (2007). Los buenos tratos a la infancia. Parentalidad, apego y resiliencia. (4 ${ }^{\mathrm{a}}$. Edición). Barcelona: Gedisa Editorial

Bené, C. Frankenberger, T., \& Nelson, S. (2015). Desing, monitoring, and evaluation of resilience intervetion: conceptual and empirical considerations. IDS working paper 2015, (459), 1-26.

Bratton, S., Ray, D., Rhine, T., \& Jones, L. (2005). The Efficacy of Play Therapy With Children: A Meta-Analytic Review of Treatment Outcomes. Professional Psychology: Research and Practice, 36(4), 376-390.

Buckner, J. C., Mezzacappa, E., \& Beardslee, W. R. (2003). Characteristics of resilient youths living in poverty: The role of selfregulatory processes. Development and Psychopathology, 15, 139-162.

Cicchetti, D., \& Rogosch, F. A. (1997). The role of self-organization in the promotion of resilience in maltreated children. Development and Psychopathology, 9, 797815.

Fall, M., Balvanz, J., \& Johnson, L. (1999). A play therapy intervention and its relationship to self-efficacy and learning behaviors. Professional School Counseling, 2(3), 194204.
Fergusson, D. M., \& Lynskey, M. T. (1996). Adolescent Resiliency to Family Adversity. Journal of Child Psychology and Psychiatry, 37(3), 281-292.

Flores, E., Cicchetti, D., \& Rogosch, F. A. (2005). Predictors of Resilience in Maltreated and Nonmaltreated Latino Children. Developmental Psychology, 41(2), 338-351. Gianino-Gadea, L. (2012). Resilience in institutionalized and non-institucionalized children. Avances en psicología, 20(2), 7990.

Góngora-Márquez, M. J. (2011). Resiliencia en niños de padres alcohólicos y su manejo a través de la Terapia de Juego. Tesis de Maestría en Psicología Aplicada. Mérida, Yucatán, México: Facultad de Psicología, Universidad Autónoma de Yucatán.

González-Arratia, L. F., Valdez-Medina, J. L., \& Zavala-Borja, Y. C. (2008). Resiliencia en adolescentes mexicanos. Enseñanza $e$ Investigación en Psicología, 13(1), 41-52.

Hou, X. L., Wang, H. Z., Guo, C., Gaskin, J., Rost, D. H., \& Wang, J. L. (2016). Psychological resilience can help combat the effect of stress on problematic social networking site usage. Personality and Individual Differences, 109, 61-66.

Kees, M., \& Rosenblum, K. (2015). Evaluation of a Psychological Health and Resilience Intervention for Military Spouses: A Pilot Study. Psychological Services, 12(3), 222230. 
Landreth, G. (2002). Play therapy: The art of the relationship. (2nd ed.). New York: BrunnerRoutledge.

Mcgloin, J. M., \& Spatz-Widom, C. (2001). Resilience among abused and neglected. Development and Psychopathology, 13, 1021-1038.

Organización Mundial de la Salud. (2013). Plan de acción sobre salud mental 2013-2020. Suiza. Recuperado de http://apps.who.int/iris/ bitstream/10665/97488/1/9789243506029 spa.pdf?ua $=1$

Pérez- Ruano, A. L. (2014). Nivel de Resiliencia en Mujeres Adolescentes Institucionalizadas en el Orfanato Valle de Los Ángeles. Tesis para obtener el grado de Licenciatura en Psicología Clínica. Guatemala, Guatemala. Facultad de Humanidades, Universidad Rafael Landívar.

Ray, D. C. (2008). Impact of play therapy on parentchild relationship stress at a mental health training setting. British Journal of Guidance \& Counselling, 36(2), 165-187

Ray, D., Bratton, S., Rhine, T., \& Jones, L. (2001). The Effectiveness of Play Therapy: Responding to the Critics. International Journal of Play Therapy, 10(1), 85-108.

Restrepo-Restrepo, C., Alpi, S. V., \& Quiceno, J. M. (2011). Resiliencia y depresión: un estudio exploratorio desde la calidad de vida en la adolescencia. Suma Psicológica, 18 (2), $1-18$.

Rocha-Arrangoiz, M. F. (2012). Psicoterapia de juego grupal focalizada con enfoque multimodal en niños institucionalizados. Eficacia en estilos atributivos. Tesis de Maestría en Psicología Aplicada. Mérida, Yucatán, México. Facultad de Psicología. Facultad de Psicología, Universidad Autónoma de Yucatán.

Sanz-Rodríguez, L. J., Gómez-García de la Pedrosa, M., Almendro-Marín, M. T., Rodríguez Campos, C., Izquierdo-Nuñez, A. M., \& Sánchez de Hoyo, P. (2009). Estructura familiar, acontecimientos vitales estresantes y psicopatología en la adolescencia. Asociación Española de Neuropsiquiatría, 29 (104), 501-521.

Steinhardt, M., \& Dolbier, C. (2008). Evaluation of a Resilience Intervention to Enhance Coping Strategies and Protective Factors and Decrease Symptomatology. Journal of American College Health, 56(4), 445-453.

Tarín, M., \& Navarro, J. J. (2006). Adolescentes en riesgo. Casos prácticos y estrategias de intervención socioeducativa. España: CCS.

Werner, E. E. (1989). High-risk children in young adulthood: A longitudinal study from birth to 32 years. American Journal of Orthopsychiatry, 59(1), 72.

Recibido el 25 de junio de 2016

Revisado el 05 de agosto de 2016 Aceptado el 03 de septiembre de 2016 